\title{
Kirche und Konstruktion sinnvollen Lebenswissens
}

\author{
von Paul M. Zulehner
}

In diesem Beitrag geht es nicht darum, ob die Kirche in Zukunft ihre Chancen nützen wird, sondern eher darum, welcher Art ihre Chancen sind. Es geht uns damit auch nicht um die Mittel, die der Kirche für ihre kommunikative Aufgabe in $\mathrm{Zu}$ kunft zur Verfügung stehen, sondern um die Frage, welche inhaltlichen Schwerpunkte ihre "Sendung" haben wird. Wir stellen diese Uberlegungen in einen wissensund religionssoziologischen Rahmen, der jeweils ohne Ankündigung auch pastoraltheologische Aspekte enthalten wird.

\section{Gesellschaft als sinnvolles Lebenswissen}

\subsection{Suche nach sinnvollem und geglücktem Leben}

Wir gehen in unseren Überlegungen davon aus, daß die Menschen auch in Zukunft nach sinnvollem und geglücktem Leben Ausschau halten. Dabei geht es ihnen darum, die entscheidenden Fragen ihres Lebens einer sinnvollen Antwort näher zu bringen. Auch in Zukunft werden dazu die Fragen nach den materiellen Gütern und ihrer gerechten Verteilung, die Frage nach der Gestaltung von Sexualität und Lebensweitergabe, die Frage nach einem friedlichen Zusammenleben der Völker in stabilen politischen Ordnungen, die Frage nach Bildung und Formung der jeweils nächsten Generation (Weitergabe angesammelten Wissensvorrates) sowie die Frage nach dem Sinn des Ganzen gehören. Aus einer anderen Perspektive könnte man sagen, es gehe um die Fragen, wie die Menschen sinnvoll umgehen mit Eros/Liebe/Sexualität, Autorität/Macht/Freiheit sowie Besitz/Vermögen'. Wir müssen auch nicht eigens hervorheben, daß die Beantwortung dieser Fragen in Zukunft nicht allein mit dem sinnvollen Leben, sondern weithin auch mit dem Uberleben der Menschheit zu tun haben wird: Man denke an die Frage der Überbevölkerung, der Unterernährung und des Hungers, des wirtschaftlichen Gefälles zwischen den verschiedenen "Welten", der Bedrohung der friedlichen Weltordnung durch technokratisch-bürokratische Regime, die Möglichkeit der Ausrottung der Zivilisation durch ABCWaffen ${ }^{2}$.

\subsection{Lebenssinn ist nicht „Eigen-Sinn"}

Es gehört zu den wichtigen humanwissenschaftlichen Erkenntnissen, daß Lebenssinn nicht "Eigen-Sinn" ist. Vielmehr nimmt ihn der einzelne jeweils von (signifikant) anderen entgegen: von seiner Herkunftsfamilie im Zuge der Primärsozialisation, von wichtigen Bezugspersonen (wie dem Ehepartner) als Erwachsener. Gewiß ist der einzelne in der Lage, das, was andere zunächst aus ihm gemacht haben, zu formen, zu modifizieren, im Zuge einer "Konversion“ auch zu verändern. Gerade Studien

Prof. Dr. Dr. Paul M. Zulehner ist o. Professor für Pastoraltheologie an der Universität Passau. 
zur Konversion ${ }^{3}$ zeigen aber wiederum, daß auch hier maßgeblich andere im Spiel sind. Kurz: Lebenssinn ist im Normalfall von anderen, von der Gemeinschaft getragen, vermittelt, nicht zuletzt auch konstruiert.

\subsection{Gesellschaftliche Konstruktion von Wirklichkeit}

So kommt folgerichtig die Wissenssoziologie auch zur These, daß Gesellschaft ein Konstruktionsvorgang von "Wirklichkeit“" ist. Im Rahmen unserer Überlegungen genügt es zu sagen: Ein Konstruktionsvorgang von "Lebenswissen“, worunter wir eben die Summe von Antworten auf die Fragen nach einer sinnvollen Gestaltung und Deutung des Lebens verstehen. Um sinnvoll zu leben, ja um überhaupt zu überleben, wird es der Menschheit in Zukunft (wie schon immer) darum gehen, unter den vorfindbaren ökonomischen, sozialen und geistigen Voraussetzungen eine entsprechende gesellschaftliche Wirklichkeit zu konstruieren, damit ein sinnvolles Lebenswissen.

\section{Kirche und Konstruktion von Lebenswissen}

Wir können nunmehr schon die Frage stellen, in welcher Art die Kirche bei dieser entscheidenden Aufgabe der Menschheit mitmachen kann. Eine Antwort auf diese Frage soll aus einer historischen Perspektive angegangen werden.

\section{1 „Christentümliche" Gesellschaft}

Die Art, nach einem Beitrag der Kirche zu fragen, ist nämlich für die Kirche relativ jung. Es gab in der "alten Welt" viele Generationen (und in der Kirche denken gewiß auch heute noch viele so: Man denke an die Traditionalisten um Lefèbvre), für die es eine Selbstverständlichkeit war, daß die wichtigsten Elemente gesellschaftlichen Lebenswissens aus dem Raum der (christlichen) Kirche(n) bezogen wurden: Dies galt gleichermaßen für den Bereich Ehe/Familie/Sexualität, aber auch die politischen Ordnungsvorstellungen, die wirtschaftlichen Modelle, die Erziehung der Kinder und der Jugendlichen, ganz besonders aber für die Fragen der Religion und der Weltdeutung. Wir haben es hier mit Gesellschaften mit einem Monopol an Lebenswissen zu tun, wobei dieses monopolisierte Lebenswissen aus dem Raum der Kirche entnommen wurde. Die Befolgung dieses Lebenswissens wurde bei den Bürgern auch mit allen zur Verfügung stehenden Mitteln durchgesetzt.

\section{2 „Säkularisierung“}

Durch tiefgreifende geistige (die Natur, auch die soziale Natur ist „machbar"), wirtschaftliche (Explosion der Naturwissenschaft, der Technik), soziale (die alte, ständisch-vorindustrielle Gesellschaft kann neue Bevölkerungsgruppen nicht mehr mit einem "Stand-Ort" versehen) und damit politische Revolutionen gerät die vorindustriell-„, christentümliche“ Gesellschaft zunehmend in Bewegung und Umwandlung. Weite Bereiche der Gesellschaft, damit aber auch des Lebens der einzelnen werden durch das überkommene gesellschaftlich-kirchliche Lebenswissen nicht mehr geformt. Man denke an die Welt der Arbeit, aber auch an die Welt der Intelligenz, in wachsendem Maße auch die Welt der Sexualität, die Welt der Jugendlichen und neuestens auch die der Frau. Das führt konsequenter Weise dazu, daß im Raum der Gesellschaft alternative "Lebenswissen" entwickelt werden, die den neuen Lebensfragen gerechter werden sollten. Entscheidend ist, daß diese neuen Lebens- 
weisheiten oft unter heftigem Protest der Kirche und damit jenseits ihrer Mauern entstanden sind. Manches Lebenswissen ist daher nicht mit innerer Notwendigkeit „anti- oder areligiös“; ihre Transzendenzarmut ist eher Ausdruck des Protests dagegen, daß kirchliche Kreise unverrückbar an alten Lebensweisheiten (unter theologisch fragwürdiger Berufung auf Jesus Christus) festgehalten haben, mit denen aber viele Menschen nicht mehr leben konnten. Man denke konkret an die Fragen der politischen Demokratie, der Religionsfreiheit, der aktiven Gestaltung der Sexualität und ihrer Funktionen, der Einschätzung der Freiheit und Autonomie sowohl des einzelnen wie auch der "weltlichen Sachbereiche“. Gewiß hat eine solche Protesthaltung auch zu einer Verkürzung mancher neuer Lebensweisheiten um die volle anthropologisch-theologische Breite geführt. Letzte Fragen wurden dann oftmals ausgeklammert oder durch neue Glaubensbekenntnisse (wie „mit dem Tod ist alles aus", „es darf keinen Gott geben") ersetzt.

\subsection{Pluralistische Gesellschaften}

Immerhin steht als vorläufiges Ergebnis dieser Entwicklung fest, daß in der Welt das überkommene christlich-kirchliche Lebenswissen, wenn überhaupt, so nur noch eines unter vielen ist: Manche politische Systeme haben ihm grundsätzlich den Kampf angesagt und sind bestrebt, an die Stelle eines christlichen Monopols von Lebenswissen ein atheistisch-kommunistisches zu setzen. Es gibt aber Vermutungen, daß diese atheistisch-kommunistischen Monopolisierungstendenzen aufgrund der veränderten ökonomischen und sozialen Bedingungen kaum auf lange Zeit durchzuhalten sind, so daß die Zukunft wahrscheinlich dem Pluralismus an Lebenswissen gehört. Lediglich durch totalitäre Systeme (ganz gleich, ob christlicher oder anderer Provenienz) wäre der Pluralismus zu überwinden. Das bedeutet aber: Die vorhin beschriebene Aufgabe der Menschen in Zukunft, unter den vorfindbaren ökonomischen, sozialen und geistigen Bedingungen sinnvolles Lebenswissen zu konstruieren, steht voraussichtlich unter der Prämisse des Pluralismus: Verschiedene Vorstellungen darüber, was für den Menschen sinnvoll ist, werden miteinander konkurrieren, jede wird von sich meinen, sinnvoller zu sein, jede wird Anstrengungen unternehmen, Menschen für sich zu gewinnen, und auch das, was an gesellschaftlichem Lebenswissen zu festen Gesetzen und Institutionen "gerinnt ${ }^{\star}$, entscheidend mitzuformen.

\subsection{Grundaufgabe der Kirche}

Damit kann schon Grundsätzliches über den Auftrag der Kirche in der Menschheit der Zukunft gesagt werden: Sie wird an der Konstruktion sinnvollen und geglückten Lebenswissens unter den Bedingungen des dritten Jahrtausends mit anderen zusammenwirken. Dabei wird sie sich auf das „Lebenswissen Jesu“ berufen. Dieses hat zu tun mit dem lebendigen Gott Jesu, dessen Zuwendung zu den Menschen, damit, daß Gott den Menschen unbedingt "angeht ${ }^{\text {“ }}$ und Gemeinschaft mit ihm sucht, daß diese Zuwendung Gottes zum Menschen, wo sie im Glauben angenommen wird, hoffnungsvoll Gemeinschaft der Menschen untereinander stiftet. Auf diese Weise erhalten die Gestaltungsaufgaben menschlicher Lebensbereiche eine Basisorientierung: Sie sind allesamt so zu gestalten, daß communio, Einheit wächst, Leben möglich, Lebensraum geschaffen wird. Gewiß hat die Kirche das für das dritte Jahrtausend erforderliche Lebenswissen heute noch nicht rezepthaft zur Hand. Das, was im Lauf der Kirchengeschichte als christlich-kirchliches Lebenswissen aus der Perspektive des Evangeliums ausgeformt wurde, behält zwar orientierenden Cha- 
rakter, zeigt, was herauskommen kann, wenn Jesu Geist in bestimmte Lebenslagen „einsickert“ und durchdringt. An den überkommenen Orientierungen kann aber noch nicht abgelesen werden, was konkret an Lebensweisheiten herauskommt, wenn der Geist Jesu in die Lebensbedingungen der kommenden Generationen „einsickert". Was aber bereits jetzt gesagt werden kann: Es wird dieses kommende christlich-kirchliche Lebenswissen in der Fluchtlinie all jener Lebenswissen liegen, die im Lauf der Geschichte im kirchlichen Gesprächszusammenhang unter Berufung auf Jesu Lebenswissen konstruiert wurden.

\section{Einzelne Aspekte kirchlichen Handelns um 2000}

Wer diesem Grundansatz zustimmt, daß die Kirche berufen ist, an der Konstruktion von sinnvollem Lebenswissen für die Menschheit im Jahr 2000 mitzuarbeiten, wird klaglos dem Weg unserer weiteren Überlegungen folgen.

\subsection{Zuwendung $\mathrm{zu}$ den Menschen}

Verwirklicht kann diese Aufgabe nur dann werden, wenn die Kirche eine hohe Solidarität mit der Menschheit hat. Die entsprechenden grundsätzlichen Beschlüsse hat das Zweite Vatikanische Konzil schon gefaßt. Hoffnung und Angste der Menschen sind auch Hoffnung und Angste der Jünger Christi, so betont das Konzil. Die Kirche wird als Zeichen und Werkzeug des Heils der Menschheit gezeichnet, damit als soziale und theologische Wirklichkeit, die nicht sich selbst lebt, sondern als Ferment des Heils anderer in der Welt ist. Nicht Auszug in ein heiles Getto (das zumal immer kleiner werden müßte), sondern dienendes Sich-der-Welt-Aussetzen wird von der Kirche und ihren Mitgliedern verlangt.

\subsection{Glaubwürdigkeit produzieren}

Als Lebensorientierung aus dem Geist Jesu wird die Kirche - mutiger als bisher vielleicht - trotz allem Respekt vor dem Wahren und Guten bei jenen, die nicht zu ihr gehören, versuchen, Menschen für sich zu gewinnen. Für sich, das heißt konkret: Für ein Leben aus dem Geist Jesu, damit für Nachfolge und Jüngerschaft. Nur so kann sie als Zeichen und Werkzeug des Heils in der Welt aufgebaut werden. In konkreten Menschen wird dann Jesu Geist in der Welt anwesend sein und in die Welt "einsidkern" können. Durch Menschen, die christlich in der modernen Welt leben, wird auch Glaubwürdigkeit hergestellt. Glaubwürdigkeit in einem doppelten Sinn: Innere Glaubwürdigkeit, indem einsichtig gemacht werden kann, daß das Lebenswissen Jesu eine verläßliche Straße zu sinnvollem und geglücktem Leben ist, äußere Glaubwürdigkeit, weil in der Kirche Menschen sind, die von sich sagen können: Wir versuchen, aus dem Geist Jesu zu leben, und machen dabei die Erfahrung, daß wir auf dem Weg zu sinnvollem und geglücktem Leben und Zusammenleben sind. Daß diese Glaubwürdigkeit mit Zeugnis zu tun hat, sei angemerkt.

\subsection{Expressive und kritische Arbeit}

Die Aufgabe der Kirche wird in erster Linie konstruktiv sein müssen. Es geht darum, (kontrasthaft) Lebensalternativen zu entwickeln, wie eben unter veränderten gesellschaftlichen Bedingungen Menschen sinnvoll, weil christlich, leben können. Das wird die Kirche aber nicht abhalten, Wahres und Gutes in Lebensmodellen anderer Menschen und Menschengruppen zu entdecken. Sie wird sich sehr oft daran erinnern, daß 
der Geist Gottes nicht die formellen Grenzen der Kirchenmitgliedschaft respektiert. So wird sie, gerade weil sie die Aufgabe, lebbares Lebenswissen zusammen mit anderen Menschen zu konstruieren, als schwierig ansieht, sehr aufmerksam das Gelungene bei anderen aufgreifen und "ausdrücklich" gutheißen. Expressiv verstärkende Arbeit wird sie leisten, wobei gar nicht immer vonnöten ist, daß das, was sie bestärkt und gutheißt, immer auch schon die Fülle des Christlichen sein muß. Im übrigen wird sie fragmentarisch Christliches auch im eigenen Haus, bei den eigenen Kirchenmitgliedern entdecken, denen es nicht immer gelingen wird, wegen der spannungsreichen Lebenssituation die Fülle des Christlichen auch in jeder Lebenslage zu leben.

Natürlich besitzt eine solche bestärkende Aufgabe bereits kritische Kraft. Wenn Gutes stark wird, wird zugleich auch weniger Gutes eingegrenzt und muß schrumpfen. Darüber hinaus wird sich die Kirche entschieden - um der Menschen willen mit den vorfindbaren Lebensweisheiten auseinandersetzen. Sie wird aufzeigen, daß sie zwar allesamt mit dem Anspruch auftreten, sinnvoll und human zu sein. Sie wird aber mit innerer Logik, aus dem Gefüge des diskutierten Systems von Lebenswissen heraus zeigen, daß auf dem gezeigten Weg das gesteckte Ziel sinnvollen und humanen Lebens nicht (voll) erreicht wird.

\subsection{Spannung zwischen Tradition und Reform}

Es ist klar, daß ein solches Agieren der Kirche heute noch keine Selbstverständlichkeit ist. Vielleicht steht ihm als stärkstes Hemmnis ein Defizit an Selbstvertrauen und pastoraler Identität entgegen. Dies führt vereinzelt zur Strategie, Ererbtes unverändert festzuhalten, jene zu sammeln, die mit der kommenden Gesellschaft in vielen Bereichen nicht zurecht kommen, die am Pluralismus als solchem zerbrechen und deshalb nach klaren Lebensanweisungen verlangen, die ganz dem entsprechen, was sie in ihrer frühen Sozialisation erlernt haben. Es ist aber unmißverständlich zu sehen, daß eine solche pastorale Strategie die Kirche für die Aufgaben der Zeit um 2000 nicht vorbereitet. Unbeschadet einer klaren christlichen Identität wird sie sich beunruhigt vor die Frage stellen, inwieweit nicht vieles an ihrem christlich-kirchlichem Lebenswissen einer versinkenden Gesellschaft angehört, obwohl es fast unentwirrbar mit dem "eigentlich Christlichen" verflochten ist. Die kritische Uberprüfung des ererbten christlich-kirchlichen Lebenswissens steht als wichtige Aufgabe an. Dabei wird es um eine doppelte Prüfung gehen: Um eine Konfrontation des Ererbten mit der Lebenswirklichkeit der Menschen, aber ebenso um eine Uberprüfung neuer Formulierungsversuche des Christlichen in neue Lebenslagen hinein am kirchlichen Gesprächszusammenhang, an der kirchlichen Tradition.

\subsection{Zukunftschancen}

Experten stehen nicht an, einer dergestalt handelnden, agierenden und nicht bloß reagierenden Kirche wachsende Zukunftschancen zu bescheinigen. Gerade die abgesättigte, durch künftige Bedrohungen aber doch zutiefst verunsicherte „säkulare Welt" beginnt, in zunehmender Dringlichkeit die Frage nach sinnvollem Leben und nach dem Sinn des Ganzen zu stellen. Mag sein, daß es heute erst kleine Gruppen sind, unter den Jugendlichen ebenso wie unter den Erwachsenen. Die Zeit scheint aber vorbei zu sein, wo man sich mit wehenden Fahnen aus traditionsträchtigen Lagern in die Reihen der Modernität begibt. Ererbtes, das heutiges Leben verstehen und gestalten hilft, genießt wachsenden Kredit. Dazu kommt, daß die Kirche offen- 
bar in wichtigen Lebensanliegen nach wie vor eine Art Monopol hat: Wo es um letztes Fundament des Lebens geht, um die Krisen des Lebens und ihre Deutung, um den Tod, um den selbstlosen Einsatz füreinander. Der Einsatz aller humanen Mittel, auch der religiösen, wird ein sinnvolles Überleben der Menschheit bringen, so behaupten die Verfasser der Berichte über die Lage der Menschheit an den Club of Rome. Sollte bei so vielen Erwartungen die Kirche keine Zukunft haben? Und wird die Kirche diese Chancen um der Menschen willen auch nützen? Es gibt viele Zeichen, die Hoffnung machen ${ }^{5}$.

\section{Anmerkungen:}

1. P. Bigo: L'Eglise et la revolution du tiers monde, Paris 1974.

2. O. K. Flechtheim: Futurologie. Der Kampf um die Zukunft, Köln 21971. Dazu auch die verschiedenen Berichte über die Lage der Menschheit an den Club of Rome.

3. M. Schbilisky: Religiöse Erfahrung und Interaktion, Stuttgart 1976.

4. P. L. Berger, T. Luckmann: Die gesellschaftliche Konstruktion der Wirklichkeit, Frankfurt 1969.

5. Zu den ganzen Uberlegungen auch: P. M. Zulehner: Einführung in den pastoralen Beruf, München 1977. - Ders., Religion nach Wahl, Wien 1974. - Ders., Heirat - Geburt Tod. Eine Pastoral zu den Lebenswenden, Wien 1976. - Ders., Säkularisicrung von Gesellschaft, Person und Religion, Wien 1973.

\section{S U M M A R Y}

The author's contribution does not examine bow the Church will contribute to communication in the year 2000 but what she will have to say. More precisely: is the Church going to say anything essential at all? He develops a short sketch of the possible structure of the Church's contribution towards dialogue in a pluralistic society, beginning with the statement that man in the future will also question the purpose of human life. Three main areas of life to come under questioning will be 1. Eros, love, sexuality. 2. Authority, power, freedom, and 3. Possession, property. The Church has to enter into dialogue with the world in these specific areas in a pluralistic society, and with a strong sense of human solidarity. The Church must offer constructive alternatives showing a meaningful forming of life. A critical appraisal of the past teachings of the Church will be required. In the areas of 'life crisis', 'death' and selfless sharing, especially, the Church has an important contribution to make towards the survival of mankind.

\section{RESUME}

La question de cette collaboration n'est pas la suivante: Comment l'Eglise apportera-t-elle sa collaboration d la communication en l'an 2000, de quel moyen se servira-t-elle? Mais: Qu'aura-t-elle d dire? Encore plus durement: Aura-t-elle, du point de vue du contenu, quelque chose d'important à communiquer? L'auteur développe en une courte esquisse le contenu structural possible d'une collaboration ecclésiastique à un dialogue pluraliste de la société. Il part du fait que les hommes continueront à se poser des questions sur le sens de la vie, et ce vraisemblement dans les trois domaines suivants: "Eros/amour/sexualité«, »autorité / puissance/ liberté", et "possession/richesse«. Dans ces domaines, l'Eglise - en reconnaissance du pluralisme et en forte solidarité avec l'homme - devra parler avec le monde, se laisser mettre en danger par lui et offrir aux hommes des alternatives constructives pour qu'ils façonnent ingénieusement leur vie. Une révision critique, progressive du 
savoir livré jusqu'ici par l'Eglise sera nécessaire. L'Eglise pourra collaborer activement à la survie de l'humanité, spécialement dans les thèmes tels que »la crise de la vie«, »la mort« et "le don total de soi

\section{RES UMEN}

Este trabajo no se pregunta: Cómo contribuirá la Iglesia a la comunicación en el año 2000, de qué medios se servirá? Se pregunta: Tendrá algo que decir? Más cáusticamente: Tendrá algo esencial que anunciar? El autor desarrolla brevemente la posible estructura de una aportación eclesial al diálogo pluralista de la sociedad. Parte del supuesto que los hombres seguirán inquiriendo sobre el sentido de la vida y probablemente sobre tres complejos: "erotismo/amor/sexualidad «, "autoridad/poder/libertad" y "propiedad/riqueza«. Reconociendo el pluralismo y en solidaridad con el hombre, la Iglesia hablará con el mundo sobre estas cuestiones, se "expondrá “ y deberá ofrecer al hombre alternativas constructivas para una configuración adecuada de la vida. Será necesaria una revision crítica de la teoría difundida hasta ahora por la Iglesia. Especialmente en temas como "crisis vital«, "muerte« y "compromiso altruísta«, la Iglesia podrá prestar una aportación importante a la supervivencia de la humanidad. 